\title{
Additional congeners of the macrolide neaumycin: structure revision and biological activity
}

\author{
Matteo Simone ${ }^{1}$, Sonia I Maffioli ${ }^{1}$, Arianna Tocchetti ${ }^{1}$, Stefano Tretter $^{1}$, Monica Cattaneo ${ }^{2}$, Ida Biunno ${ }^{2}$, \\ Eleonora Gaspari ${ }^{1}$ and Stefano Donadio ${ }^{1}$
}

The Journal of Antibiotics (2015) 68, 406-408; doi:10.1038/ja.2014.168; published online 14 January 2015

\section{INTRODUCTION}

Analysis of whole-genomic sequences of actinomycetes has consistently indicated that each strain has the potential to produce several distinct families of specialized metabolites. ${ }^{1}$ Whereas many of these molecules are not produced under normal laboratory conditions, a strong emphasis on bioassay-guided screening might have also resulted in compounds with modest activity having been overlooked in past studies. ${ }^{2}$ Re-investigation of already-described strains often leads to the detection of previously unreported metabolites, ${ }^{3,4}$ without the need to use special triggers to activate expression of cryptic clusters. The macrolide neaumycin (1) was recently isolated from the soil actinomycete Streptomyces sp. NEAU-x211 and reported to consist of a 30membered macrolide featuring a unique internal diester bridge (Figure 1a). ${ }^{5}$ No significant antimicrobial or cytotoxic activity was detected up to $1 \mu \mathrm{M}$. While investigating Actinoplanes sp. ATCC $33076^{6}$ the producer of ramoplanin, a glycolipodepsipeptide antibiotic discovered in 1984 and highly active against multidrug-resistant Grampositive bacteria, ${ }^{7,8}$ we observed that the strain also produced a family of unrelated compounds, one of which was established to be identical to neaumycin (1; Figure 1).

\section{ISOLATION AND STRUCTURE ELUCIDATION}

Here, we report the isolation and characterization of two novel neaumycin congeners, which led to a structural revision of the parent compound. While optimizing the conditions for ramoplanin production by Actinoplanes sp. ATCC 33076, we observed that the strain produced, under several different growth conditions, lipophilic HPLC peaks distinct from the ramoplanin complex. Upon adjusting the HPLC conditions (see Supplementary Information), these peaks could be resolved and one of them was identical by UV and MS to 1, hereafter designated as neaumycin A (Figure 1). ${ }^{5} \mathrm{NMR}$ analysis on the purified powder confirmed the identity showing ${ }^{1} \mathrm{H}$ and ${ }^{13} \mathrm{C}$ signals undistinguishable (Supplementary Figures S16 and S17 and Supplementary Table S4) from that of the already-published neaumycin A. The other lipophilic peaks, designated neaumycin B (2), C (3), D and E (Supplementary Figure S2), possess the same UV spectrum as 1. After cultivating Actinoplanes sp. ATCC 33076 in medium AF-1 maximal production of the neaumycin complex was observed after $96 \mathrm{~h}$ with an approximate titer of $7 \mathrm{mgl}^{-1}$ (Supplementary Figure S3) showing comparable amounts of 1 and 2 and lower amounts of $\mathbf{3}$. The neaumycin complex was purified (SI) leading to the isolation of $\mathbf{1}, \mathbf{2}$ and $\mathbf{3}$ in sufficient amounts for structure elucidation. Compound 2, having retention time of 18.72 min, showed $\mathrm{m} / \mathrm{z}$ signals at 875.6 and 897.6 , corresponding to the [M $+\mathrm{H}]^{+}$and $[\mathrm{M}+\mathrm{Na}]^{+}$ions, respectively, highlighting a 112-amu difference from 1. NMR analysis of 2 highlighted the absence of signals originating from the 3 -methylfumaric residue of 1 . Yet, a further dehydration must be have occurred in 2 to account for the observed mass difference between 1 and 2. Upon NMR analysis only the methine group at position 13 showed a significant change in ${ }^{1} \mathrm{H}$ chemical shift, from $\delta 4.95$ in $\mathbf{1}$ to 3.48 in 2 , as expected from the absence of esterification with the fumaric moiety; smaller changes were also observed for the protons located at the vicinal positions 11, 12 and 14. No further relevant changes in chemical shifts were observed between 1 and $\mathbf{2}$ (Supplementary Table S1). In particular, the methine group at position 27, which is esterified in structure 1a with the fumaric residue, maintains the same high chemical shift (4.88 and $4.8 \mathrm{ppm}$ in 1 and 2, respectively), suggesting that its diagnostic low field value is actually due to a chemical environment different from fumarate esterification, whereas a clear HMBC correlation between $\mathrm{H}-27$ and the carboxyl signal at $166.1 \mathrm{ppm}$ indicates its esterification with position 1 (Supplementary Figure S10). Moreover, the diagnostic differences in the chemical shifts of the diastereoisomeric methylene hydrogens at positions $24,28,30,31$ and 32 in 1, which indicate a zone of low flexibility, are superimposable to the corresponding signals in 2. This observation suggests that the rigidity of this part of the molecule is not because of the annulation resulting from diesterification of the fumaric moiety, but results instead from a different element of rigidity common to both molecules. Upon MS analysis, we observed that, whereas 1 was equally detected under negative and positive ionization, 2 could only be observed by positive ionization, suggesting that the two congeners have different ionizable groups. This was confirmed by testing the chemical reactivities of $\mathbf{1}$ and 2 toward amines in the presence of a condensing agent: whereas the latter 
a

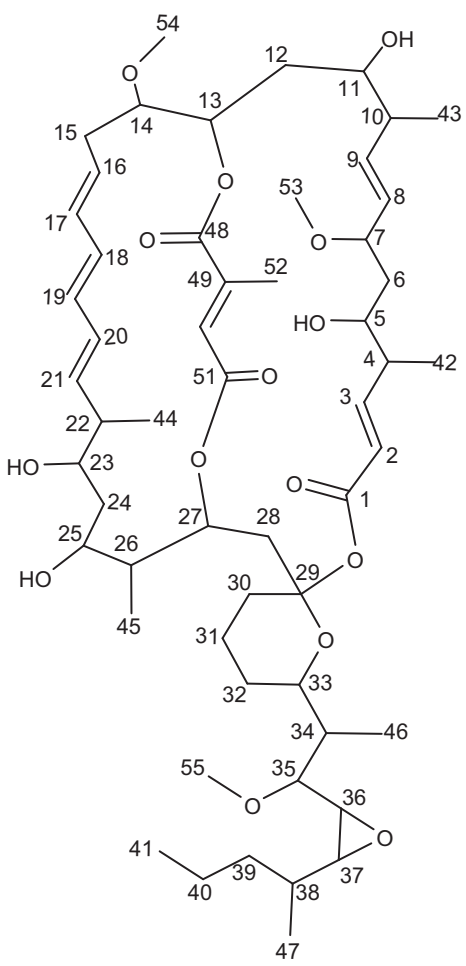

b

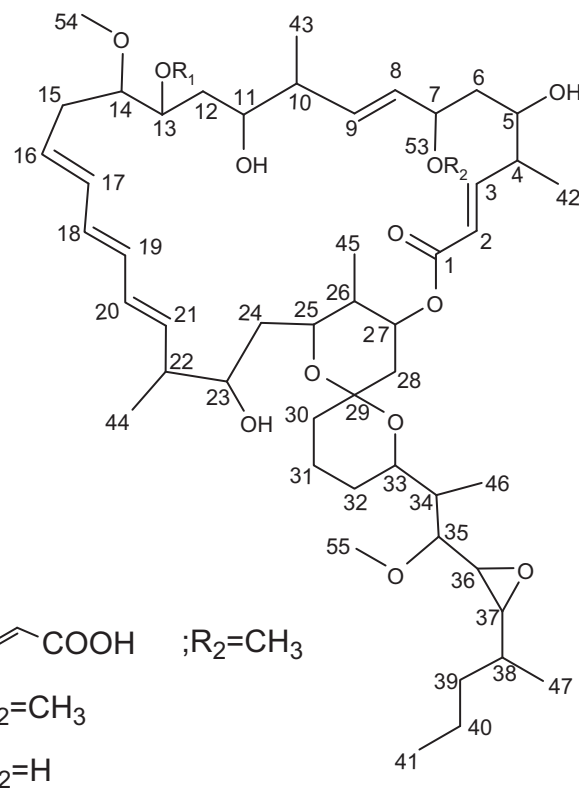<smiles></smiles>

Figure 1 Previously published (a) and revised (b) chemical structure of neaumycins.

compound remained unchanged, 1 yielded the amidated compound 4 having $m / z$ 1071.7 $[\mathrm{M}+\mathrm{H}]^{+}$(Supplementary Figure S4). Thus, 1 contains a free carboxyl group and the fumaric residue must be linked to position 13 only, whereas position 27 must be involved in a rigid moiety common to $\mathbf{1}$ and $\mathbf{2}$. On the basis of the above observations, we propose that $\mathbf{1}$ actually has the structure $\mathbf{1 b}$ (Figure 1 ).

Accordingly, neaumycin A consists of a spiroketal-containing macrocycle with a 3-methylfumaric moiety linked to position 13 . The 28-membered macrocycle is formed by esterification of the carboxyl group at position 1 with the hydroxyl at position 27, consistent with the C-27 chemical shift remaining unmodified in 2 . The spiroketal moiety accounts for the necessary dehydration, results in agreement with the C-29 chemical shift and explains the rigid system observed for the methylenes at positions 24, 28, 30, 31 and 32, which remains unaltered in $\mathbf{2}$. Further confirmation of structure $\mathbf{1 b}$ came after alkaline hydrolysis of $\mathbf{1}$, which readily generated $\mathbf{2}$ as the first product. The other purified congener neaumycin C (3), with retention time of $15.79 \mathrm{~min}$ and $\mathrm{m} / \mathrm{z} 861.6$ for $[\mathrm{M}+\mathrm{H}]^{+}$and 883.9 $[\mathrm{M}+\mathrm{Na}]^{+}$, resulted closely related to 2 , by comparison of the ${ }^{1} \mathrm{H}$ and ${ }^{13} \mathrm{C}$ NMR spectra that clearly indicate the absence of the methyl group at position 7 in 2 (Supplementary Tables S1 and S2). Whereas NMR spectroscopic data suggest that compounds 1, 2 and 3 share the same stereochemistry, it remains to be determined whether the spiroketal-containing fragment C25-C34 has the relative configuration proposed by Shen and co-workers ${ }^{9}$ or whether it resembles the structurally related molecules 21-hydroxyoligomycin A, neomaclafungins ${ }^{10}$ and rutamycin. ${ }^{11}$ We also observed additional congeners related to $\mathbf{1}$, named neaumycin $\mathrm{D}$ and $\mathrm{E}$, with signals at $\mathrm{m} / \mathrm{z}$ $868[\mathrm{M}+\mathrm{Na}]^{+}$and $897.8[\mathrm{M}+\mathrm{Na}]^{+}$and retention times of 17.12 and $18.07 \mathrm{~min}$, respectively. Their $\mathrm{m} / \mathrm{z}$ values suggest that all these congeners lack the mesaconate moiety, which is probably one of the late steps during neaumycin biosynthesis. Moreover, neaumycin D, more lipophilic and lighter for $16 \mathrm{amu}$ in respect of neaumycin $\mathrm{C}$, probably lacks one of the hydroxyl groups. However, the small amounts of purified congeners D and E prevented elucidation of their structures.

\section{BIOLOGICAL ACTIVITY}

Little cytotoxic activity was observed for $\mathbf{1}$ and 2 at 1 or $10 \mu \mathrm{M}$ against two different cell lines. However, at $100 \mu \mathrm{M}$ both compounds fully inhibited cell proliferation. This result is consistent with previous work in which no cytotoxic activity of 1 was observed at $1 \mu \mathrm{M}$. Compound 1 showed a modest but measurable activity against a few Gram-positive bacteria, with MICs of $64 \mu \mathrm{g} \mathrm{ml}^{-1}$ observed against Staphylococcus aureus and Streptococcus pneumoniae and $16 \mu \mathrm{g} \mathrm{ml}^{-1}$ against S. pyogenes. No activity (MIC>128 $\mu \mathrm{g} \mathrm{ml}^{-1}$ ) was observed against these strains using 2. Both compounds were inactive against Gram-negative bacteria $\left(\mathrm{MIC}=178 \mu \mathrm{g} \mathrm{ml}^{-1}\right)$ or Candida albicans $\left(\mathrm{MIC}>128 \mu \mathrm{g} \mathrm{ml}^{-1}\right)$. The observation that $\mathbf{1}$ and related compounds were produced, under most conditions, together with ramoplanin, prompted us to investigate whether the combination of ramoplanin with either 1 or 2 would result in enhanced antibacterial activity. We thus employed a methicillin-resistant Staphylococcus aureus strain in a checkerboard dilution assay. The data are reported in Figure 2. Remarkably, the presence of either $\mathbf{1}$ or $\mathbf{2}$ significantly reduced the MIC of ramoplanin in a dose-dependent manner from $4 \mu \mathrm{g} \mathrm{ml}^{-1}$ in the absence of neaumycin to $0.015 \mu \mathrm{g} \mathrm{ml}^{-1}$ in the presence of either $4 \mu \mathrm{g} \mathrm{ml}^{-1} \mathbf{1}$ or $16 \mu \mathrm{g} \mathrm{ml}^{-1} 2$. Thus, as little as $4 \mu \mathrm{g} \mathrm{ml}^{-1} \mathbf{1}$ (that is, a concentration $1 / 16$ th the MIC of $\mathbf{1}$ ) was able to reduce the ramoplanin MIC by two orders of magnitude. A similar behavior was observed with 2. No enhancement of the MIC was observed when similar checkerboard assays were performed with vancomycin. After 


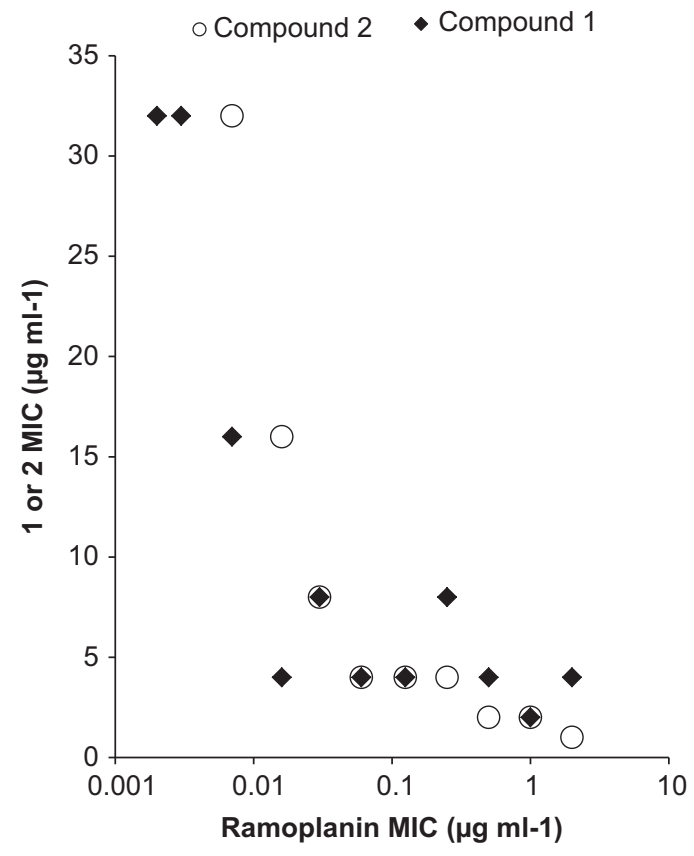

Figure 2 Interaction of compounds $\mathbf{1}$ and $\mathbf{2}$ with ramoplanin against MRSA.

structural revision, $\mathbf{1}$ bears a strong resemblance to the macrocyclic systems found in actinomycete metabolites such as maclafungin, ${ }^{12}$ neomaclafungins, ${ }^{10}$ oligomycin ${ }^{13}$ and rutamycin. ${ }^{11}$ Despite this similarity, the structural originality of neaumycin noticed by Shen and coworkers ${ }^{5}$ persists in the following features: (i) a previously unreported 28-membered, spiroketal-containing macrocycle; (ii) a conjugated system containing three, instead of the usual two double bonds; (iii) a long lateral chain at position 33 carrying a unique epoxy group; and (iv) a 3-methylfumaric residue, which separates $\mathbf{1}$ from other metabolites carrying an unsubstitued fumaric moiety, such as hygrolidin, ${ }^{14}$ AH-758, ${ }^{15}$ PD-118.576 ${ }^{16}$ and bafilomycin. ${ }^{17}$ The oligomycin-type macrolides reported above display a wide variety of activities, including anticancer, antifungal, nematocidal, insecticidal and immunosuppressive effects. ${ }^{10}$ In particular, oligomycin, apoptolidin, cytovaricin, ossamycin and venturicidin have been widely characterized as inhibitors of oxidative phosphorylation. ${ }^{18}$ The description of an additional but unusual member might provide further insights into structure activity relationships within this class of macrocycles. As a further advancement to the previous study, ${ }^{5}$ we also report that $\mathbf{1}$ is produced as a complex of related congeners, one of which has been characterized as likely biosynthetic precursors of neaumycin lacking the fumarate moiety. Finally, we have shown that a modest antibacterial or cytotoxic activity can be observed for $\mathbf{1}$ and $\mathbf{2}$. The significant enhancement of ramoplanin activity in the presence of either $\mathbf{1}$ or $\mathbf{2}$ is striking. However, as this enhancement is seen with a large molar excess of $\mathbf{1}$ or $\mathbf{2}$, we refrain from considering it a synergistic effect. Further investigations will be required to establish the nature of this enhancement. We would like to make one additional observation from the present study. Because of its potent antibacterial activity, ramoplanin has been investigated as a potential drug for many years, reaching a phase 3 clinical study for the prevention of infections by vancomycin-resistant enterococci. ${ }^{19}$ Consequently, the Actinoplanes
ATCC 33076 strain has been thoroughly investigated in industrial laboratories. Nonetheless, the neaumycin complex must have been systematically overlooked or deemed devoid of interest over all those years. $6,20,21$ We believe that this is not an exception and new compounds can be identified also by examining other 'thoroughly investigated' strains.

\section{ACKNOWLEDGEMENTS}

This work was partially supported by grant from Italian MIUR and Regione Lombardia. We are grateful to CPC Biotech (Burago di Molgora, Monza Brianza) for fermentation work, and to Ben Shen and Margherita Sosio for expert advice.

\section{CONFLICT OF INTEREST}

MS, SIM, AT, EG and SD are employees of and/or hold shares of Naicons Srl

1 Baltz, R. H. Renaissance in antibacterial discovery from actinomycetes. Curr. Opin. Pharmacol. 8, 557-563 (2008)

2 Monciardini, P., Iorio, M., Maffioli, S., Sosio, M. \& Donadio, S. Discovering new bioactive molecules from microbial sources. Microb. Biotechnol. 7, 209-220 (2014).

3 Aigle, B. et al. Genome mining of Streptomyces ambofaciens. J. Ind. Microbiol. Biotechnol. 41, 251-263 (2014).

4 Mazzei, E., Iorio, M., Maffioli, S. I., Sosio, M. \& Donadio, S. Characterization of madurastatin C1, a novel siderophore from Actinomadura sp. J. Antibiot. (Tokyo) 65 267-269 (2012).

5 Huang, S.-X. et al. Neaumycin: a new macrolide from Streptomyces sp. NEAU-x211. Org. Lett. 14, 1254-1257 (2012).

6 Cavalleri, B., Pagani, H., Volpe, G., Selva, E. \& Parenti, F. A-16686, A new antibiotic from actinoplanes I. fermentation, isolation and premilinary physico-chemical characteristics. J. Antibiot. (Tokyo) 37, 309-317 (1984).

7 Rolston, K. V. et al. In-vitro activity of ramoplanin (a novel lipoglycopeptide) vancomycin, and teicoplanin against gram-positive clinical isolates from cancer patients. J. Antimicrob. Chemother. 38, 265-269 (1996)

8 Citron, D. M. et al. In vitro activities of ramoplanin, teicoplanin, vancomycin, linezolid, bacitracin, and four other antimicrobials against intestinal anaerobic bacteria. Antimicrob. Agents Chemother. 47, 2334-2348 (2003).

9 Wagenaar, M. M., Williamson, R. T., Ho, D. M. \& Carter, G. T. Structure and absolute stereochemistry of 21-hydroxyoligomycin A. J. Nat. Prod. 70, 367-371 (2007).

10 Sato, S., Iwata, F., Yamada, S. \& Katayama, M. Neomaclafungins A-I: oligomycin-class macrolides from a marine-derived actinomycete. J. Nat. Prod. 75, 1974-1982 (2012).

11 Arnoux, B. et al. X-Ray structure of the antibiotic rutamycin. Chem. Commun. 318-319 (1978).

12 Mukhopadhyay, T. et al. Maclafungin, a new antifungal macrocyclic lactone from Actinomycete sp. Y-8521050. Tetrahedron 54, 13621-13628 (1998).

13 Visser, J. et al. Production and isolation of the antibiotic, oligomycin. J. Biochem. Microbiol. Technol. Eng. 2, 31-48 (1960).

14 Seto, H., Akao, H., Furihata, K. \& Ōtake, N. The structure of a new antibiotic, hygrolidin. Tetrahedron Lett. 23, 2667-2670 (1982).

15 Uyeda, M., Kondo, K. I., Ito, A., Yokomizo, K. \& Kido, Y. A new antiherpetic agent produced by Streptomyces sp. strain no. 758. J. Antibiot. (Tokyo) 48, 1234-1239 (1995).

16 Hatfield, G. M., Woodard, R. W. \& Son, J.-K. Isolation and structure determination of new macrolide antibiotics. J. Nat. Prod. 55, 753-759 (1992).

17 Werner, G., Hagenmaier, H., Drautz, H., Baumgartner, A. \& Zahner, H. Metabolic products of microorganisms. 224. bafilomycins, a new group of macrolide antibiotics production, isolation, chemical structure and biological activity. J. Antibiot. (Tokyo) 37, 110-117 (1984).

18 Hong, S. \& Pedersen, P. L. ATP synthase and the actions of inhibitors utilized to study its roles in human health, disease, and other scientific areas. Microbiol. Mol. Biol. Rev. 72, 590-641 (2008).

19 Montecalvo, M. A. Ramoplanin: a novel antimicrobial agent with the potential to prevent vancomycin-resistant enterococcal infection in high-risk patients. J. Antimicrob. Chemother. 51, 31-35 (2003).

20 Gastaldo, L. et al. Isolation, structure determination and biological activity of A-16686 factors $A^{\prime} 1, A^{\prime} 2$ and $A^{\prime} 3$ glycolipodepsipeptide antibiotics. J. Ind. Microbiol. 11, 13-18 (1992).

21 Pan, H. X. et al. Production of ramoplanin analogues by genetic engineering of Actinoplanes sp. J. Biotechnol Lett. 35, 1685-1692 (2013). 